\title{
Modelos de Competitividad para Destinos Turísticos en el Marco de la Sostenibilidad
}

\section{Competitiveness Models for Tourist Destinations into the Sustainability Context}

\author{
Rosana Mara Mazaro * \\ Doutora em Ciências Empresariais pela Universidade de Barcelona, Espanha. \\ Professora do DEPAD/UFRN, Natal/RN, Brasil.
}

Giovani Varzin

Doutorando em Sociologia pela Universidade de Barcelona, Espanha.

Professor do DEPAD/UFRN, Natal/RN, Brasil.

*Endereço: Rua do Maruim, 772, Natal/RN, 59122-010. E-mail: rosanamazaro@uol.com.br 


\section{RESUMEN}

Los avances en la comprensión de los factores que determinan la competitividad de los destinos turísticos y principalmente la inclusión de la sostenibilidad como indicador de desempeño han ocasionado distintos modelos teóricos de representación del sistema turístico de una localidad. El artículo explora el contenido de cuatro de estos modelos que interpretan la dinámica del sistema turístico en base a factores del nuevo contexto competitivo influenciado por los condicionantes sostenibles. El reto es identificar, conceptuar y caracterizar los principales factores que están presentes en cada modelo y por medio de análisis comparativo establecer sus propiedades comunes, en base a categorías de atributos previamente definidos. El análisis permite señalar que estos modelos convergen en la indicación de los factores y atributos del paradigma de la sostenibilidad para los destinos turísticos como elementos estratégicos hacia su planeamiento, organización y gestión.

Palabras claves: competitividad turística; gestión de destinos; desarrollo sostenible.

\section{Abstract}

The step forward into the comprehension which determines the competitive context of the tourist routes and, especially, the insertion of the sustainability as a performance indicator are orienting distinct theoretical models of local tourist system representation. This article explores the content of four of these models which have as a common characteristic to interpret the dynamics of the tourist system under the light of a new competitive context inevitably influenced by sustainable conditioners. The separate analyses allows to identify, conceptualize and characterize the main determining factors of the competitive success in each model, and the confront between them enables to confirm a convergent context and scenery in the indication of the sustainability paradigm attributes for the tourist routes as strategic elements for its planning, organization and management.

Key words: tourist sustainability; competitiveness of tourist destinies. 


\section{Hacia una Nueva Comprensión del Turismo}

De acuerdo con las previsiones de la Organización Mundial del Turismo [OMT], el rápido crecimiento de las corrientes turísticas en los últimos decenios continuará hasta llegar a ser la actividad comercial más importante del planeta, con un número de transacciones mayor que el de la industria automotriz y la del petróleo. Esto supone por lo pronto una creciente presión competitiva en aras del turismo.

Para hacer frente a los desafíos implícitos en este escenario, los ensayos científicos de Bosch Campubrí, Marco, Cabado y Riera (1998), Crouch y Ritchie (1999), Genest y Legg (2003), Goeldner, Ritchie y McIntosh (2000), Mihalic (2000), Pine y Gilmore (1999), Ritchie y Crouch $(1999,2003)$ y los esfuerzos de la OMT (2000) y World Travel and Tourism Council [WTTC] (2004) en promocionar el desarrollo del turismo en clave sostenible realzan la necesidad de se avanzar hacia modelos de interpretación del sistema turístico de mayor complejidad, estructurado a partir de nuevos valores y con un soporte intenso de conocimientos científicos y tecnológicos; modelos no sólo orientados a atenuar impactos ambientales, sino también a instaurar un nuevo umbral del desarrollo, en el que más allá de perseguir la viabilidad de la actividad turística, busque satisfacer las necesidades de las comunidades, de los turistas y de quienes operan negocios con criterios de sustentabilidad.

Concomitantemente se manifiesta la necesidad de los agentes del turismo, tanto privados como públicos, abordar la gestión de los flujos turísticos y la organización de los destinos turísticos desde una perspectiva coherente, integrada y cualitativa, en vista de ser capaces de supervisar y gestionar la totalidad de un sistema donde los participantes se benefician del turismo y contribuyen de diversas formas a sufragar los costos que genera.

El ritmo al que aumentan y varían las motivaciones y necesidades de los turistas se traduce en una compleja segmentación de la demanda turística. Por consiguiente, es necesario diversificar aún más la oferta y personalizar el producto turístico, tanto en su conjunto como en sus numerosos componentes, cada uno de los cuales participa en la formación de la experiencia vivida por el turista, especialmente en los casos donde los recursos endógenos del destino constituyen la esencia de la oferta turística.

En efecto, los destinos turísticos deben proponer tantas alternativas como requiera la segmentación de la demanda turística y tienen que definir una serie de estrategias de desarrollo del turismo en vista de crear valor para la totalidad del sistema. La 
cadena que lleva a la creación de valor debe incluir a todos los participantes de los destinos turísticos, desde las actividades turísticas locales, hasta la demanda turística y la comunidad de acogida, su identidad y sus recursos naturales y culturales, para convertirse en la cadena de creación de valor del destino turístico.

La amplitud y significado del turismo en cuanto actividad de impactos multidimensionales para una determinada localidad se modelan en el concepto de destino turístico articulado por Valls (1998):

Espacio geográfico determinado, con rasgos propios de clima, raíces, infraestructuras y servicios, y con cierta capacidad administrativa para desarrollar instrumentos comunes de planificación, que adquiere centralidad atrayendo a turistas mediante productos perfectamente estructurados y adaptados a las satisfacciones buscadas, gracias a la puesta en valor y ordenación de los atractivos disponibles; dotado de una marca y que se comercializa teniendo en cuenta su carácter integral (p. 34).

El concepto brinda la comprensión de los atributos implicados en la gestión integral de un destino turístico y fija de manera concisa los principales condicionantes de la competitividad global. Desde luego, siempre y cuando haya referencia a la gestión de destinos, se estará considerando todo el abanico de variables y atributos aquí implicados. Esta observación se hace necesaria para el mejor entendimiento del modelo de análisis de la competitividad de los destinos bajo los criterios de la sostenibilidad turística comentados a continuación.

\section{Destinos Turísticos: Oportunidades en Clave a la SOSTENIBILIDAD}

Diferentes manifestaciones en el mundo han posibilitado comprobar que la existencia misma del turismo puede imponer una presión a la capacidad del medio que utiliza y que la actividad turística puede degradar las bases mismas sobre las que reposa su prosperidad. La fuerte presión socioambiental provocada por el incremento de las actividades turísticas y el deterioro de los recursos en diferentes destinos turísticos del mundo han provocado la convergencia entre los temas acerca del desarrollo del turismo y el paradigma de la sostenibilidad.

La OMT (1993) define como turismo sostenible al que se orienta a satisfacer las necesidades de los turistas actuales, generando ingresos y bienestar social en el destino, pero conservando al mismo tiempo los recursos y garantizando la continuidad de las ofertas y de las actividades a largo plazo. Son formulaciones 
basadas en la búsqueda de la compatibilidad integral entre preservación y desarrollo, y suponen los primeros signos del escenario futuro para la construcción de las estrategias del sector, que tiene en las exigencias ambientales y culturales los principales reclamos competitivos.

Para cumplir con ello, es esencial la estructuración de una oferta turística local que actúe a medio y largo plazo como importante factor de desarrollo. Igualmente es necesario que posibilite aprovechar los potenciales que ofrece el territorio, brindando nuevas modalidades basadas en el uso equilibrado y sostenido, así como una utilización y administración de los recursos turísticos bajo una práctica integral y flexible conforme a la dinámica competitiva.

Turismo sostenible introduce al nuevo concepto de turismo y describe su aplicación, tanto en la política turística como en las iniciativas del propio sector, mediante capítulos específicos para administraciones, empresas y turistas. Desde el punto de vista de este trabajo y en rigor constituye un concepto que implica, en cualquier caso, la utilización de un modelo de gestión de los recursos desde la perspectiva de su proyección hacia el futuro, esto es, un modelo planificado (Bosch Campubrí et al., 1998).

Para evaluar el grado de desarrollo alcanzado por un plan turístico, los modelos teóricos consideran sostenible el turismo cuando: respeta (no daña) y valora al medio ambiente natural, cultural, humano y social, donde se manifiesta; aporta beneficios financieros directos importantes y/o recursos para el desarrollo de la comunidad (infraestructuras, educación, herramientas de organización, etc.); promueve la participación local en el proyecto, o promueve un proceso participativo de los miembros de la comunidad en el proceso de su propio desarrollo como seres humanos; integra las herramientas de evaluación y de seguimiento permanentes para garantizar controle sobre los impactos.

El concepto e implementación de un turismo sostenible implica necesariamente interdependencia de la institución pública con su política de acción, medio ambiente, población local y visitantes que se encuentran en una situación de interacción permanente. A pesar de su contenido ambiguo, lo que se pretende es discutir lo que hay de válido, serio y objetivo en el ideal del desarrollo sustentable. Aunque desde recientemente se observa una integración de la política turística en una estrategia más amplia de desarrollo sostenible, este no puede ser entendido sólo como opción deliberada de una estrategia de institucionalización de la problemática ambiental en el ámbito de las organizaciones internacionales y de los gobiernos nacionales (Godfrey, 1998).

Mas allá de colocar la necesidad de prudencia ecológica en la agenda internacional, las orientaciones sostenibles deben interpretar-se como guiones a 
los gestores del turismo para hacer frente a la competitividad turística y al compromiso con la calidad de vida de las localidades. Para que la sustentabilidad adquiera una estatura real, que incida de una manera determinante en los procesos sociales, se requiere el desarrollo de una nueva generación de valores y conceptos, de metodologías e instrumentos, todos ellos congruentes entre sí.

La visión de futuro constituye un elemento importante para la diferenciación entre los destinos turísticos. Los principios que orientan la visión en el marco del turismo sostenible están volcados principalmente en limitar el impacto de sus actividades sobre la biósfera, mantener el patrimonio biológico, utilizar los recursos sin agotarlos, procurar un reparto equitativo de costos y beneficios, promover tecnologías limpias, utilizar la política económica para mantener los recursos naturales y decidir con criterio previsor y transectorial.

La compatibilidad entre las potencialidades e impactos del turismo es resultado de la visión de futuro emprendida por la gestión local, que orienta y condiciona las acciones del desarrollo turístico. Así, comprender la visión y la elección estratégica permite plantearse el futuro y la capacidad competitiva del destino. Estos elementos son comprensibles cuando están declarados formalmente y son profusamente comunicados a los públicos interesados en el turismo local, pues es precisamente el proceso de planeamiento participativo el que imprime y fija el modelo de desarrollo que debe orientar las estrategias y acciones turísticas en el destino.

En otras palabras, la competitividad turística exige tratar la sostenibilidad como condición estratégica en la organización y gestión de los destinos y sobre todo en la composición y supervaloración de la oferta. El desafío está en la elección de un modelo de oferta turística que asuma el impacto del turismo en la economía local, reconfigurando una nueva estructura de su sistema productivo y de gestión, pero que al mismo tiempo ha de esforzarse en preservar su identidad social y cultural como un valor fundamental.

Algunos elementos de estos desafíos parecen generalizables cuando el caso es la implementación de prácticas sostenibles en el turismo. El primero de ellos es dimensionar y combinar diferentes capacidades de carga. La esencia es el balance, el equilibrio entre las capacidades de carga ecológica, física, social, económica, experimental y gubernamental. Hay que administrar la competencia intensiva por el uso del suelo y del espacio, considerando que hay diversos intereses y formas de explotación y potenciación de los recursos turísticos.

Además, atentar para los cambios en los hábitos de los consumidores, tanto en lo que se relaciona a los factores de decisión, como a los relacionados al consumo y disfrute de la oferta en el destino y todavía combatir la especulación verde, relacionada a las prácticas sostenibles superficiales con el propósito exclusivo de 
aprovechar la vulnerabilidad del mercado y que pueden comprometer la imagen del destino hacia el futuro.

Sin embargo, la capacidad de comprender que las prácticas sostenibles ofrecen un largo rango de oportunidades para el sector turístico ha garantizado que unos pocos tomen posición de vanguardia con larga ventaja competitiva en atención a segmentos de mercado sensibles a tales estímulos y con perspectiva de crecimiento continuo, a escala que se amplia la conciencia por la diversidad y en donde la búsqueda por la calidad de vida vuelve el ícono del milenio (Bosch Campubrí et al., 1998).

Los vínculos directos que existen entre las actividades turísticas y la calidad del medio ambiente demuestran que el sector tiene mucho que ofrecer y que ganar convirtiéndose en líder del desarrollo sostenible (Bosch Campubrí et al., 1998; Hudson \& Miller, 2003; Mihalic, 2000). Esto es particularmente cierto en los países de turismo emergente, en los que el sector turístico constituye tanto un sostén al desarrollo como un vehículo para el rescate y mantenimiento de la identidad cultural. Asimismo, este concepto es particularmente válido en los ambientes frágiles, donde el desarrollo del sector turístico puede constituir una fuerza significativa en el mantenimiento o mismo para mejorar el medio ambiente.

La integración de la política turística y el fomento del turismo sostenible, en torno a lo que podríamos denominar planes de ordenación del turismo, se sitúan entre los requisitos más urgentes la aprobación de directrices que tutelen la implantación de la oferta turística, el control de los procesos urbanizadores, la gestión del paisaje, así como el control sobre actividades contaminantes, habitualmente ajenas a la legislación turística. No se trata sólo de cualificar, modernizar y renovar destinos, como se ha hecho hasta ahora, sino que hay que establecer limitaciones al crecimiento continuado de la oferta y acometer una planificación integrada de espacios y destinos turísticos que permita seleccionar la tipología de oferta, los modelos de implantación y sus exigencias infraestructurales.

\section{Modelos de Competitividad para Destinos Turísticos en Clave A LA SOSTENIBILIDAD}

Los avances en la comprensión de los factores que determinan la competitividad de los destinos turísticos y sobre todo la inclusión de la sostenibilidad como indicador de desempeño, han ocasionado distintos modelos teóricos que intentan representar el complejo sistema donde se plasma el turismo con toda su implicada red de interacciones. 
En términos teóricos y conceptuales, los principales modelos están representados a seguir. Cada un de ellos es sucintamente definido y analizado bajo una evaluación crítica de sus propiedades y en base a atributos como motivaciones para su desarrollo, objetivos propios del modelo y fundamentos tanto teóricos como prácticos.

\section{Modelos de Desarrollo Turístico e Impactos Ambientales - las Nuevas Coordenadas}

La interpretación de cómo sigue la marcha del desarrollo turístico, hacia dónde puede llegar y lo qué se puede lograr con las diferentes formas de implementárselo, ha sido un esfuerzo continuo de los científicos en los últimos años. De acuerdo con Bosch Campubrí et al. (1998), los modelos de desarrollo turísticos se resumen en dos tipos básicos: intensivo continuo y extensivo puntual.

- El intensivo continuo sigue la pauta del modelo de desarrollo turístico tradicional cuyas características corresponden a la densificación y congestión urbanística, deterioro del paisaje y del ambiente, ofertas estandarizadas, desconexión entre los espacios, desaprovechamiento de los recursos y de fuertes impactos negativos sobre el medioambiente.

- El extensivo puntual corresponde a los modelos alternativos de desarrollo turístico, invariablemente basados en un desarrollo horizontal y equilibrado que provoca una imagen de alta calidad en la recuperación y aprovechamiento de los recursos y del paisaje. "Se caracteriza por una importante prevención de la contaminación y por una preocupación por la protección de los espacios naturales, mientras se incorporan los recursos culturales" (p. 87).

Para los autores el primero corresponde al modelo de desarrollo turístico tradicional; el segundo es alzado como modelo alternativo, en respuesta a las características del nuevo modelo turístico y a las nuevas coordenadas de la gestión del espacio turístico.

Los factores y características del modelo turístico pueden ser entendidos a través de cuatro grandes fuerzas: consumidores, tecnología, producción y gestión, cada una de las cuales con implicaciones múltiplas en su dimensión de análisis. Estas fuerzas están determinadas por un conjunto global y competitivo muy singular y único en la historia, en donde factores como flexibilidad, segmentación y preocupación medioambiental se han puesto como condicionantes del desarrollo turístico.

El foco de análisis de los autores es el medioambiente y bajo este prisma apuntan 
como las nuevas coordenadas de la gestión del espacio turístico, los postulados que siguen (Bosch Campubrí et al., 1998):

“...creciente sensibilidad ante la degradación paisajística y los costos medioambientales del crecimiento turístico" (p. 88);

. "protección de espacios naturales, como franjas litorales, islas, ríos, montañas, flora, etc." (p. 88);

- "potenciación de los recursos naturales como oferta alternativa y que permita recuperar el territorio como atractivo esencial: los senderos, caminos de ronda, antiguos trazados ferroviarios, etc." (p. 88);

. "incorporación de recursos culturales en la oferta turística: museos, parques arqueológicos, festivales de música o gastronomía típicos, itinerarios monumentales o históricos, etc." (p. 88).

Aunque las consideraciones de los autores no hagan referencia explicita a la dimensión económica de la sostenibilidad turística y que los principales parámetros de soporte al modelo imparten de observaciones realizadas en destinos urbanos de países de Europa, como es el caso de España, los elementos y factores involucrados en la interpretación de los autores suelen ser los mismos para cualquier otra localidad turística preocupada con la competitividad global, esté ella en medio al concurrido mediterráneo o en los rincones mas lejanos de Latinoamérica.

La aproximación es derivada de los estudios y contribuciones de los autores en sus actividades académicas y en consultoría hacia el tratamiento de temas emergentes en turismo como es el medioambiente, una de las principales variables de influencia sobre las decisiones hacia el desarrollo sostenible para el turismo. En cuanto a los fundamentos científicos de la proposición, aunque basados en larga experiencia en planeamiento de destinos y productos turísticos, la interpretación aquí reproducida tiene carácter fundamentalmente teórico conceptual.

\section{Modelo del Sistema Interfuncional Integrado de la Competitividad en Destinos Turísticos [SIIC]}

A partir de Porter (1990), que define los cuatros atributos que promueven - o impiden - la creación de la ventaja competitiva nacional, Toledo, Valdez y Polero (1998) proponen un modelo teórico referencial que orienta metodológicamente los análisis y estudios sobre la integración de los elementos del sistema turístico y sus interrelaciones en un cluster. "Con un enfoque holístico y a partir de la teoría general de los sistemas, se considera que el sector de turismo, específicamente un cluster turístico, es un sistema abierto homeostático con equifinalidad, configurado para alcanzar la competitividad sustentable" (p. 14). 
En la percepción de los autores, un cluster presenta características intrínsecas de la actual fase de la globalización, pues integra con un objetivo común las diversas instituciones intersectoriales que surgen de la nueva relación pública y privada: entidades mixtas, gubernamentales, no gubernamentales, universidades, de capacitación de la fuerza de trabajo, incubadoras de proyectos, asociaciones comerciales de clase, agencias de información, órganos de investigación y de soporte técnico.

Un cluster está formado por empresas independientes unidas informalmente para poder ofrecer al cliente ventajas diferenciales. Según Musa (2001), constituye una forma alternativa de organizar la cadena de valor a través de redes de alianzas y sociedades entre las empresas de forma tal de aumentar y facilitar la flexibilidad para superar la rígida competencia tradicional.

El modelo del Sistema Interfuncional Integrado de la Competitividad en Destinos Turísticos [SIIC] que apoyan está formado por cinco factores y sus interrelaciones en el sistema turístico: los dos primeros, la masa crítica y la naturaleza de la demanda tienen las características de crear condiciones básicas para el desarrollo del cluster. Estos ofrecen una configuración de partida para su planificación estratégica. Los tres elementos que completan el modelo son los que mejoran la ventaja competitiva del cluster y se concretan por medio de las estrategias competitivas, las estrategias cooperativas y las estrategias de relacionamiento con el cliente.

La masa crítica es el conjunto de los factores básicos necesarios para el desarrollo del destino turístico. Abarca la integración de los recursos turísticos (tierra, agua, geografía, clima, localización); humanos (calidad, capacitación, identidad cultural histórica, participación social); de capital (incentivos, inversiones, riesgos); infraestructura (sistema de transporte, telecomunicaciones, facilidades a la inversión); de conocimientos (técnicos científicos, de las universidades, de investigación y desarrollo, de las asociaciones comerciales, las organizaciones no gubernamentales, de los órganos rectores y de fomento); superestructura (organización institucional intersectorial, relaciones privadas y públicas, entidades de clase, relaciones con países emisores); seguridad (órganos de certificación de la sustentabilidad, sistema de apoyo al turista, garantía de libre disfrute de la estada y del uso de sus atractivos con seguridad).

La naturaleza de la demanda, los autores interpretan por medio de atributos derivados de la naturaleza, deseos y expectativas del consumidor turístico, del potencial de la demanda y de los padrones de calidad o sofisticación de los segmentos abordados.

La estrategia competitiva que, según Porter (1993), debe basarse en una rivalidad interna vigorosa, sin restricciones y en igualdad de condiciones para 
competir, resulta en un ambiente fértil en el cual las empresas del cluster pueden crecer competitivamente. Este tipo de competición exige estrategias actualizadas y constante introducción de innovaciones para satisfacer mejor las necesidades del cliente. Un grupo de rivales internos fuertes en el cluster constituye un ingrediente esencial para el suceso competitivo en este tipo de configuraciones.

El éxito internacional del cluster exige un ambiente local que coloque las empresas en padrones internacionales de calidad de servicios y que, en lugar de inhibir, facilite alcanzar estos padrones de competitividad. El estilo de competir dentro del cluster debe ser pujante para mejor satisfacer al cliente sobre la base de la calidad y diferenciación, mejorando así su identidad por su competitividad.

El proceso de agrupamiento geográfico que es intrínseco al sector turístico fundamenta la estrategia de cooperación. Esta concentración en polos o destinos con atractivos turísticos no siempre ha estado acompañada por el fomento de las interrelaciones y alianzas de los agentes del sistema. Se ha utilizado el nombre genérico de cluster turístico como sinónimo de destino o polo, aunque no opere con características de los clusters ya madurados en otros sectores económicos en la actual fase de la globalización.

El cluster, como una configuración especial que surge en el contexto de la globalización, tiene como propiedad principal el estrechamiento de las relaciones entre las organizaciones para crear una red de sinergias; su objetivo es el desarrollo de estrategias competitivas conjuntas, creando y entregando mayor valor al mercado, por medio de relaciones cooperativas entre empresas independientes que tienen un mismo objetivo, aunque compitan entre sí.

El modelo propuesto sugiere que la diferencia real entre cluster turístico y polo turístico tradicional está en que, además de considerar los elementos de partida (masa crítica y naturaleza de la demanda), imprescindibles para desenvolver al polo, un cluster turístico busca su posición competitiva a escala global a través de un espacio tridimensional, que combina estrategias competitivas, estrategias cooperativas y estrategias de relaciones con el cliente que, con sus insumos o inputs (necesidades, deseos y expectativas), optimizan la configuración del sistema, retroalimentándolo con el proceso simultáneo de producción, consumo y evaluación. Es este un proceso cuya ventaja competitiva es alcanzada por el vector resultante de las tres estrategias.

El modelo no está caracterizado por las dimensiones de la sostenibilidad y representa una adaptación del modelo de Porter (1995), para sostener la proposición de los clusters turísticos como estrategia más efectiva para la competitividad. Como se ha dicho en el primer párrafo, la proposición es de alcance teórico referencial y de carácter metodológico abstracto. 


\section{Clasificación de Destinaciones Turísticas - The Premier-ranked Tourist Destinations}

The Premier-ranked Tourist Destinations Workbook (Genest \& Legg, 2003) es una metodología desarrollada con el apoyo del gobierno de Canadá para servir como herramienta para mensurar el status competitivo o situación de mercado de una destinación turística. La herramienta sirve de instrumento a servicio de la gestión y su intento es involucrar todos los elementos y variables críticas del contexto competitivo para destinaciones turísticas. Las informaciones reveladas suelen servir de base para el planeamiento estratégico y para el desarrollo del turismo como actividad económica de una localidad.

Para los autores, la metodología esta estructurada de forma que haga posible:

- capturar los atributos/factores/condiciones considerados necesarios para que una destinación turística sea percibida como en posición competitiva privilegiada;

- identificar debilidades y amenazas que puedan ser administradas o contornadas;

- identificar fuerzas y oportunidades que puedan ser utilizadas para crear ventajas competitivas sustentables;

- promover la acción coordinada entre los diferentes sectores, favoreciendo el consenso sobre las prioridades para acción futura.

La evaluación consiste en un proceso analítico sistemático de las condiciones de competitividad del destino por medio de tres dimensiones de análisis: de producto, de desempeño y de futuro, incluyendo medidas de sustentabilidad y de interrelaciones de los factores.

La dimensión producto evalúa las condiciones de la oferta turística del destino y su capacidad de proporcionar experiencias impactantes al visitante, a través de los siguientes atributos: recursos y atractivos esenciales; calidad y masa crítica; satisfacción y valor; accesibilidad; y equipos y servicios.

La dimensión desempeño evalúa los resultados del sector y si el destino tiene éxito en proporcionar experiencias de calidad a los visitantes, por medio de atributos e indicadores del flujo turístico, ocupación y rentabilidad, y reconocimiento crítico e imagen.

La dimensión futuro evalúa la capacidad del destino de mantenerse competitivo a lo largo del tiempo, analizando los factores y atributos de las estrategias de marketing, innovación y renovación y sustentabilidad local. 
Para cada un de los factores y atributos están indicados criterios y medidas que sustentan la interpretación. La metodología está especificada en un guía de trabajo donde están descritas desde la forma de su aplicación, hasta los requisitos y criterios para interpretación de sus resultados. Adquiere así un carácter eminentemente práctico y de aplicación a la realidad de cualquier destinación interesada en evaluar sus condiciones competitivas en un determinado contexto.

Sin embargo, la proposición aunque imparta un bien delimitado estado de la cuestión y de embasamiento teórico de referencia consistente para la elección de los criterios y dimensiones de análisis, concentra su foco en elementos competitivos de la oferta y de mercadeo. En otras palabras, la perspectiva y alcance del instrumento están enfocados en los componentes del marketing turístico, sin vincularse directamente a los condicionantes del desarrollo de la localidad, como puede ser la política y gestión del turismo local, y también sin profundizarse en los impactos más amplios de las actividades turísticas, ya sean positivos o negativos. En definitiva, la proposición no está desarrollada para evaluar competitividad en clave a sostenibilidad, aunque contemple atributos e indicadores fundamentales de la aproximación, más bien sirve para conocer lo que hace que una destinación logre alcanzar una posición de liderazgo competitivo en un determinado contexto.

En lo que toca al alcance de sus resultados, la metodología ha sido puesta a prueba en la región que comprende la cuesta sur de Ontario, Canadá, más específicamente en la Bahía Georgian, una de las más populares destinaciones para las playas en verano y para las estaciones de esquí en invierno, combinando una significativa herencia cultural y de eventos.

Hay que considerar la etapa de desarrollo y los condicionantes del ambiente competitivo en Canadá en donde sus destinaciones se encuentran en avanzada etapa de lucidez política en el tema turismo. La metodología supone un elevado grado de comprensión del turismo local por parte de los evaluadores, igualmente de un sentido critico desproveído de apegos ideológicos.

De acuerdo con los autores, el teste confirmó la utilidad del modelo y su habilidad en identificar las fuerzas y debilidades de una región, dificultades en la gestión y toda una amplitud de aspectos que están presentes en cada una de sus dimensiones de análisis. Sobre todo argumentan que la metodología debe ser comprendida como un instrumento que capacita a las destinaciones interesadas en impartir y efectuar por si mismas una autoevaluación de las condiciones actuales y futuras de competitividad en el contexto del turismo. 


\section{Modelo Conceptual de Competitividad y Sostenibilidad para Destinaciones Turísticas}

El modelo presentado por Ritchie y Crouch (2003) es fruto de sus estudios e investigaciones en turismo a lo largo del último decenio motivados por el incremento de la competitividad en el mundo del turismo. Desde la primera presentación pública del modelo en 1993, los autores han promovido la continua revisión y adaptación de las variables que componen el modelo para los nuevos imperativos del contex to competitivo determinados por elementos socioculturales, económicos, antropológicos, tecnológicos, comportamentales, medioambientales, entre aquellos de mayor impacto (Crouch \& Ritchie, 1999).

Tal modelo está representado en la Figura 1.

\section{Figura 1: Modelo Representativo de Competitividad y Sostenibilidad para Destinaciones Turísticas}

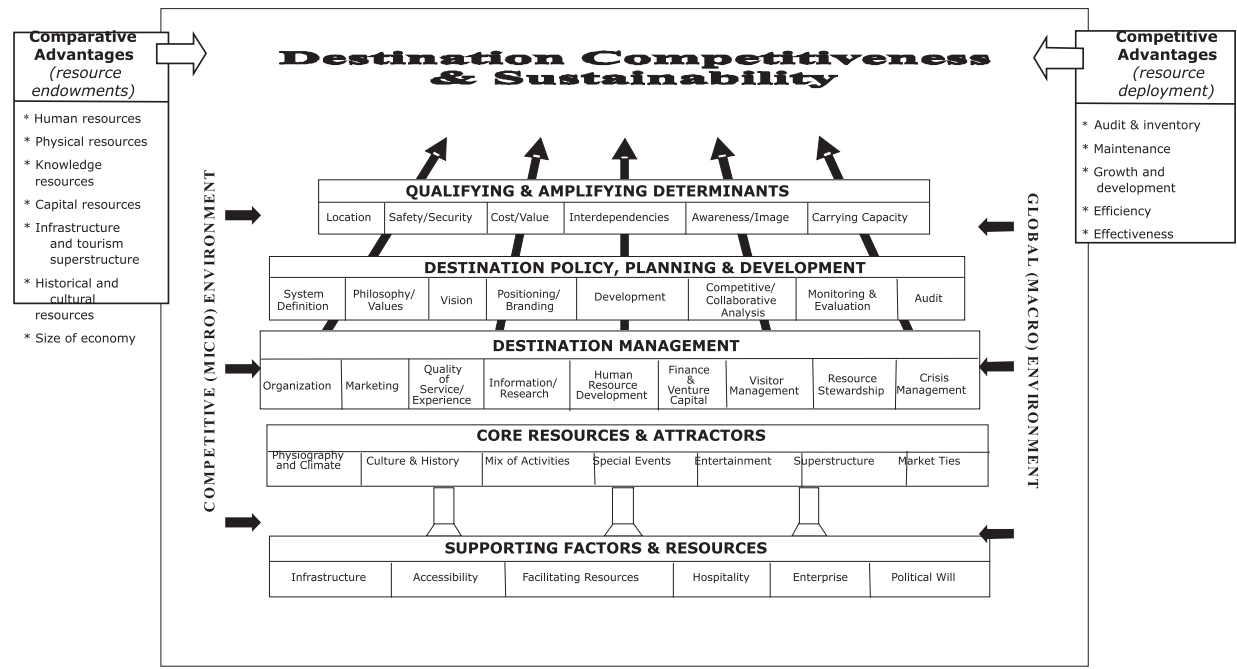

Fuente: Ritchie y Crouch (2003).

En la evaluación de los autores, el cambio más significativo entre anteriores presentaciones del modelo y su interpretación actual está en que los factores política turística y administración del destino antes tratados en separado y de manera independiente, pero en el contexto de la competitividad actual se los consideran de mutua y estrecha interdependencia y se los interpretan como elementos responsables por la necesaria conexión entre el deseado - planos - y las acciones de que se ocupa a diario una destinación - programas. Para ellos esta es una categoría indispensable para que se engendren las condiciones bajo las cuales el turismo pueda desarrollarse de manera apropiada y sostenible (Ritchie \& Crouch, 2003). 
Como queda enseñado en su representación gráfica, el modelo de Destinos Competitivos y Sostenibilidad [DCS] es casi exhaustivo en el apunte de dimensiones y atributos que completan lo que se comprende por sistema turístico y por las variables que condicionan su competitividad. Los principales factores de impacto están clasificados en cinco categorías de elementos estratégicos como se resume a continuación.

Tomando por referencia el significado de ventaja comparativa y ventaja competitiva en el contexto del turismo, el esquema alude a los recursos innatos y atractivos esenciales como elementos primarios de apelo de la destinación y que constituyen elementos clave para la motivación y persuasión de visitantes y para estudios prospectivos sobre demanda y mercadeo.

Para Crouch y Ritchie (1999, p. 147) estos recursos están distribuidos en otras subcategorías: clima y naturaleza, cultura y historia, etnia, actividades, eventos especiales y superestructura turística. En el juicio de los autores, el rango de actividades ofrecidas es un elemento crítico del apelo de una destinación y sobre el cual los gestores tienen extensiva influencia y control. Esto es un aspecto relevante para la tesis y será rescatado en el capítulo de proposición.

Importante es la justificación cuando incluyen la categoría superestructura como un de los factores primarios de atracción. Ellos argumentan que si por un lado es reconocido el hecho del visitante no elegir un destino sólo por la cama y la comida, más bien por la llamada en clave a los atractivos que sostiene, por otro lado, mirando hacia ejemplos emblemáticos de destinaciones como Las Vegas y Miami en Norteamérica, Dubai en Arabia; Barcelona y París, en Europa, se percibe que, en conformidad con la calidad de estos recursos, pueden ser ellos mismos parte importante de la atracción, o sea los componentes de superestructura turística pueden ser proyectados de forma que sean ellos los propios factores primarios de atracción para los visitantes.

Una segunda grande categoría está clasificada en el modelo de Ritchie y Crouch (2003), como factores y recursos de soporte. Estos, como el nombre ya indica, están relacionados a los elementos que posibilitan que las actividades y disfrute de los servicios turísticos se concreticen, pues entre ellos están relacionados la infraestructura, servicios básicos y complementarios, accesibilidad y capacidad emprendedora y empresarial en la destinación.

Estas dos grandes categorías de factores primarios constituyen los pilares sobre los cuales se puede fundamentar el turismo en un determinado destino, pues sirven de materia prima o inputs para la tercera grande categoría denominada por Ritchie y Crouch (2003, p. 9), como administración del destino. En ella están vinculadas la forma de organización de la destinación y los procesos de 
toma de decisiones sobre marketing y posición competitiva, recursos humanos y capacitación, investigación y desarrollo, captación de capital e inversiones, entre tantos otros. La misión de la administración es realzar el atractivo originado en el rango de recursos primario y de soporte, fortalecer la calidad y la efectividad y por fin adaptar las recursos de la destinación a los determinantes competitivos.

De acuerdo con lo antes comentado, en esta última versión del modelo acrecientan una nueva categoría de factores relacionados a política, planeamiento y desarrollo del destino cuyos componentes se refieren a las grandes orientaciones para el turismo, como la filosofía, visión y directrices para el desarrollo, lo que resultará en decisiones sobre posición y nivel de coompetición $^{(1)}$, además de las necesarias medidas de auditoría, monitoreo y evaluación permanentes en lo concerniente al desempeño e impactos del turismo en la destinación.

Según la visión de los autores, una comprensión del significado de política de turismo requiere un análisis a través de tres dimensiones: la estructura de la política, que establece un esquema de interrelación dentro del cual los contenidos específicos pueden ser formulados; un proceso sistemático de formulación política que involucra los diferentes y complementarios grupos de interés en turismo. Este proceso es influenciado por la dinámica e interacción de los actores y factores del sistema, una vez que presupone una base mínima de consenso entre los agentes para que definan la estructura y contenido de esta misma política para el turismo de una determinada destinación. Una conceptualización de estructura política del turismo más detallada está en Goeldner et al. (2000).

Como quinta y ultima macrocategoría de factores que integran al modelo conceptual están apuntados los calificadores y potenciadores determinantes. Algunos de estos factores están relacionados a la localización geográfica y capacidad de carga de la destinación que se caracterizan por ser involuntarios y respecto a los cuales, a la gestión solamente cabe potenciar; a la vez, otros factores como la seguridad, la imagen, costo/valor y niveles de interdependencia de las destinaciones están condicionadas por estrategias definidas y manejadas por los gestores del turismo local en conjunto con los demás agentes y apostadores.

O sea, unos pocos factores determinantes no son pasibles de intervención estratégica por la administración local, en cuanto la mayoría de ellos pueden y deben ser tratados como elementos capaces de integrar las características propias del lugar con las implementadas en la destinación a causa de la intervención y organización política y administrativa a fin de alcanzar niveles de calidad capaces de proyectar la destinación en la disputa por un lugar al sol.

Tal condición representa el medio en el cual el turismo se desarrolla; en el 
modelo está segmentado por un de nivel macro, en referencia a las variables incontrolables globales, como puede ser la tecnología, la economía, el medioambiente, las convenciones y diplomacias, la política, etc.; y un de nivel micro, en referencia a variables del ambiente más cercano entendido como aquello que caracteriza las condiciones competitivas de una destinación turística y es, en última instancia, lo que diferencia una de las otras de acuerdo a los autores.

El modelo es conceptual; no es predictivo ni tampoco causal; más bien es una propuesta en sus primarias conjeturas. El modelo es relativamente abstracto en su formato actual y no permite aplicaciones o generalizaciones empíricas específicas. Está mejor definido como una teoría o un tópico, en conformidad a la topología de Neuman (Crouch \& Ritchie, 1999, p. 146).

El mayor mérito del modelo es ser exhaustivo en el apunte de las dimensiones y factores implicados en el sistema turístico y en la interpretación del contexto y condicionantes de la competitividad para destinos en clave a la sostenibilidad. Para esta tesis representa el marco teórico referencial más completo y más bien fundamentado fundamentado en términos de madurez, profundidad y amplitud en la lectura del medio y condiciones de impacto sobre el turismo como sector y sobre las actividades que de ello se originan y que deben ser observadas por aquellos que decidieren seguir sus rutas.

\section{A Modo de Conclusión}

La virtud de un enfoque de planificación estratégica radica en proporcionar un marco genérico en el que tienen cabida distintas combinaciones de iniciativas públicas y privadas, con la clara identificación de los roles y responsabilidades de cada parte, favoreciendo un análisis integrado para la gestión del destino, a través de un conjunto de factores habilitados para mediar la performance de los destinos turísticos.

De forma general se puede decir que el estado de la cuestión respecto al tema tratado en este artículo ubica en la etapa de sedimentación de los postulados generales que fundamentan las aproximaciones de ellos derivadas, que demuestran estar conformadas por medio de amplio debate y articulación entre diferentes actores y sectores.

El Cuadro 1 presenta una síntesis comparativa de las características principales de los modelos examinados que facilitan la comprensión de los elementos que orientarán el análisis en este estudio. 





Tras el estudio y análisis de los modelos queda la sensación que las dimensiones, elementos, mecanismos e indicadores de evaluación de las condiciones de competitividad están señaladas en sus representaciones, y se destaca el hecho de los factores efectivamente determinantes de estas condiciones, aunque con nominaciones distintas, están contemplados en todos los formatos de interpretación teórica del conjunto de circunstancias, indicando las fuerzas y las debilidades para enfrentarse al contexto de la competencia entre destinos.

Asimismo, queda revelado un cierto nivel de consenso entre los científicos en lo que toca a los factores de consideración estratégica para la gestión sostenible del turismo local. Entretanto la ubicación y las interconexiones de y entre cada factor en la interpretación propia del sistema turístico de cada modelo, es lo que los hacen diferentes entre sí.

En cuanto a las orientaciones generales para el desarrollo turístico, las evidencias indican que cada vez más destinaciones están adoptando una perspectiva estratégica que combine intereses de marketing con una visión de desarrollo turístico; una preocupación con los impactos multidimensionales del turismo, en contra una preocupación estrechamente económica; un mirar hacia el horizonte de largo plazo por encima de los efectos de curto plazo; en definitiva, una orientación en clave al turismo sostenible.

Como bien enuncia Ritchie y Crouch (2003), cuyo modelo es el que mejor representa el sistema turístico en el marco del desarrollo sostenible, las destinaciones que están orientadas por sus principios hacia el planeamiento y gestión reconocen que competitividad es ilusoria sin sostenibilidad. En la visión de los autores, que corresponde integralmente al pensamiento del presente análisis, los términos competitividad y sostenibilidad para el turismo es hoy una tautología.

\section{Artigo recebido em 01.04.2006. Aprovado em 07.04.2007.}

\section{NotA}

${ }^{1}$ El término es escrito con dos os para representar la necesaria cooperación entre los agentes del turismo local a fin de lograr niveles efectivos de competición como destinación turística sostenible (Crouch \& Ritchie, 1999). 


\section{Bibliografía}

Bosch Campubrí, R.,

Marco, L. P.,

Cabado, J. S., \&

Riera, F. V. (1998).

Turismo e meio ambiente. Madrid: Centro de Estudios Ramon Areces.

Crouch, G. I., \&

Ritchie, B. J. R. (1999).

Tourism, competitiveness, and societal prosperity. Journal of Business Research, 44(3), 137-152.

Genest, J., \&

Legg, D. (2003).

Premier-ranked tourist destinations: development of a framework for analysis and its self-guided workbook. Ontario. Recuperado em 17 março, 2004, de http:// www.tourism.gov.on.ca/english/ rese a c h/pdf/self - guidedworkbook.pdf

Go, F., \&

Govers, R. (2000).

Integrated quality management for tourist destinations: a European perspective on achieving competitiveness. Tourism Management, 21(1), 79-88.

Godfrey, K. B. (1998).

Attitudes towards sustainable tourism in the UK: a view from local government. Tourism Management, 19(3), 213-224.
Goeldner, C.,

Ritchie, J. R., \&

McIntosh, R. W. (2000).

Tourism: principles, practices, philosophies (8th ed.). Toronto: John Wiley \& Sons, Inc.

Hudson, S., \&

Miller, G. A. (2003).

The responsible marketing of tourism: the case of Canadian Mountain Holidays. Tourism Management, 24(2), 82-95.

Mihalic, T. (2000).

Environmental management of a tourist destination: a factor of tourism competitiveness.

Tourism

Management, 21(1), 65-78.

Musa, F. (2001).

Uma visão de conjunto. CLUSTER Revista Brasileira de Competitividade, (Edição Especial), 194-207.

Organización Mundial del Turismo. (1993).

Sustainable tourism development: guide for local planners. Madrid: Author.

Organización Mundial del Turismo. (2000).

Turismo panorama 2020: nuevas previsions. Madrid: Author.

Pine, B. J., \&

Gilmore, J. H. (1999).

The experience economy: work is theater \& every business a stage. Boston, Massachusetts: Harvard Business School Press. 
Pilgram, J. J. (1990).

Sustainable tourism: policy considerations. Journal of Touris Studies, 1(5), 2-9.

Porter, M. E. (1990).

The competitive advantage of nations. New York: The Free Press.

Porter, M. E. (1993).

A vantagem competitiva das nações. Rio de Janeiro: Campus.

Porter, M. E. (1995).

Green and competitive. Harvard Business Review, 73(5), 120-134.

Ritchie, J. R. B., \&

Crouch, G. I. (1999).

Crafting a value-driven vision for a national tourism treasure. Tourism Management, 20(3), 273-282.
Ritchie, J. R. B., \&

Crouch, G. I. (2003).

The competitive destination. Wallingford: CAB International Publishing.

Toledo, G. L.,

Valdés, J. A., \&

Pollero, A. C. (1998).

Empresas turísticas en ambiente globalizado: marketing y competitividad. Estudio de casos de clusters turísticos. São Paulo: FEA/ USP.

Valls, J. F. (1998).

Cambio de concepto en la gestión turística del municipio. Estudios de Gestión Turística, 7, 3-7.

World Travel and Tourism Council. (2004). The WTTC report: blueprint for new tourism. Recuperado em 17 março, 2004, de http://www.wttc.org/ blueprint/WTTCBlueprintFinal.pdf 